\title{
Special Issues
}

Since 1975, the Annals of Biomedical Engineering has had at least 35 special issues or special sections within an issue. The special issues consist of articles detailing scientific advances in a review or original research format. They have been organized around particular themes or topics, or originated as papers presented at annual scientific meetings of interest to BMES members, or emerged from discussions that occurred during those meetings. In its early years, most of $A B M E$ 's special issues came out of scientific meetings and symposia. Examples of issues that were based on meetings include the following: 2005 "Bone Fluid Flow: Organ to Cell, Lab Bench to Bedside, On Earth and In Space" (5th International Bone Fluid Flow Workshop held in Cleveland, OH, 2003); 2001 "Biomechanics" (Augustburg Conference of Advanced Science, Augustburg, Germany, 1999); 1989 "Biomechanics and Biomaterials" (5th Southern Biomedical Engineering Conference held in Shreveport, LA, 1986); 1981 "Engineers and the Respiratory System" (34th Annual Conference on Engineering in Medicine and Biology, 1981).

Thematic special issues have become more common over time. Especially under Larry McIntire's leadership there was an emphasis on identifying areas of wide interest to our readers. A few noteworthy examples include: 2009 on Nanobioengineering; 2008 on Cellular Biorheology and Biomechanics; and 2002 on Biomechanical Approaches to Atherosclerosis.

In the past one-and-a-half years, the Annals has published two special issues: In June 2010, Michael Detamore, serving as the Guest Editor, compiled an issue that thematically centered on interfaces and interfacial bioengineering. Though when it comes to impact of particular issues one year is too short of a time period, the results thus far indicate that the papers in that special issue have been well-received, as evidenced by the more than 40 citations and hundreds of downloads that have already been made. The April 2011 issue was our second special issue, based on the 11th Annual UC Systemwide Bioengineering Symposium, held 17-19 June 2010 and attended by over 300 people. The vast majority of these papers, overseen by Guest Editors Angie Louie and Dan Kamei, were published as open access due to an agreement between the University of California and the publisher. We expect that the papers, equally distributed between original research and reviews, will be widely downloaded and cited, not only because they are excellent peer-reviewed contributions but also because of their open access nature. One of these papers has already been downloaded 250 times over a period of 3 weeks, and is one of $A B M E$ 's most downloaded papers over the past 30 days.

The Annals editorial office is working with some internationally respected biomedical engineers to continue publishing special issues. Already in the works, the following special issues should appear in the next few months: Biophotonics in Bioengineering; Glycomechanics: Sugar coating blood cell-endothelial interactions in shear flow; and nano/micro mechanical systems for lab-on-chip applications.

It is no secret that special issues are important to the Annals, as they help our field by their concentrated foci, be it thematic or regional. So, we will use this forum to remind our readers that we will entertain proposals for special issues that are either based on a topic or a sub-field, or on a scientific BME/BIOE meeting. The meetings can be thematic or they can be regional. If other US states, or countries, or geographic regions hold scientifically strong special meetings, we would urge the scientific organizers to consider submitting a proposal to us. These meetings are important for sharing and disseminating ideas, generating discussions, and forming new ideas.

In particular, we would like to ask you to consider submitting proposals for special issues that focus on particular aspects of biomedical engineering. Please consider established areas that merit an in-depth examination via both original and review articles. But, please also consider emerging areas of large interest, especially those multidisciplinary ones that use nonexistent or blurred scientific boundaries. We will work with the guest editors to ensure that every submission undergoes our usual, rigorous review process.

Respectfully submitted,

K. A. Athanasiou

University of California, Davis

Electronic mail: athanasiou@ucdavis.edu 